\title{
Thrombotic thrombocytopenic purpura in a case of mycoplasma pneumoniae
}

\author{
P N Rajakrishna ${ }^{1}$, D L B Dassanayake ${ }^{2}$, A W M Wazil ${ }^{1}$, D G S Alahakoon ${ }^{3}$, A Siribaddana $^{2}$ \\ Journal of the Ceylon College of Physicians, 2010, 41, 89-91
}

\begin{abstract}
A 25-year-old man had four days of fever, vomiting, diarrhea and shortness of breath. On admission he had evidence of severe pneumonia with hypotension not responding to fluid challenges, needing ICU care with cardiorespiratory support. He developed thrombocytopenia, anemia, right sided weakness and renal impairment with normal coagulation profile and high LDH. Mycoplasma antibody titer rose with the disease course. We made a diagnosis of thrombotic thrombocytopenic purpura secondary to mycoplasma pneumoniae. With plasma exchange therapy, he made a remarkable recovery. This case illustrates a rare association of mycoplasma pneumoniae and thrombotic thrombocytopenic purpura, and the importance of initiation of appropriate therapy when it is suspected.
\end{abstract}

\section{Background}

The classic description of thrombotic thrombocytopenic purpura (TTP) comprises the pentad of features; microangiopathic hemolytic anemia (MAHA), consumptive thrombo-cytopenia, neurological abnormalities, renal impairment and fever ${ }^{1}$. TTP first came to medical literature in 1925, described by Moschowitz ${ }^{2}$, which remains an uncommon syndrome, with a high mortality rate. With the advent of plasma exchange the survival has improved to $60 \%$ to $90 \%$ in an acute attack $^{3}$. This has led to the change of diagnosis criteria of TTP. According to the American Association of Blood Banks, the American Society for Apheresis, and the British Committee for Standards in Hematology, only the presence of former two manifestations without any apparent alternative cause is sufficient to diagnose TTP and is an indication to start plasma exchange therapy ${ }^{1}$.

\footnotetext{
${ }^{1}$ Nephrology and Transplant Unit,

${ }^{2}$ Chest Unit,

${ }^{3}$ Medical Unit, Teaching Hospital, Kandy.
}

TTP syndromes are described in association with pregnancy, autoimmune disorders, pro-drome of bloody diarrhea, immune and dose dependent drug toxicity and hemopoietic stem - cell transplantation ${ }^{4,5}$. Association with infections is sparsely reported and there were only few reports of the association with myco-plasma pneumoniae on a PubMed search ${ }^{6,7}$. Mycoplasma pneumonia, the second com-monest cause of community acquired pneumonia, is renowned for its peculiar extra-respiratory associations ${ }^{8}$.

\section{Case report}

A previously well 25-year-old mechanic, presented to a medical ward with a four day history of fever, loose stools, vomiting and shortness of breath. On admission he had a temperature of $39.4^{\circ} \mathrm{C}$. He was cyanosed and in shock with unrecordable blood pressure and had scatted coarse crepitations in both lung fields. With the working diagnosis of septic shock secondary to pneumonia, he was resuscitated with fluids, inotropes and broad spectrum antibiotics were given to cover atypical pneumonias.

He was transferred to Intensive Care Unit (ICU) as he was rapidly deteriorating and needed ventilation. His chest $x$ ray revealed bilateral alveolar shadows and had a white cell count of $30 \times 10^{9} / \mathrm{L}$ with a neutrophil leukocytosis. His erythrocyte sedimentation rate was $78 \mathrm{~mm}$ for $1 \mathrm{st}$ hour, with a CRP of $38 \mathrm{mg} / \mathrm{dl}$. Serology for mycoplasma, H1N1, dengue and leptospirosis were negative. Sputum for AFB was negative. LE cells were not detected. Blood, urine and endotracheal tube cultures were negative. On the second day of ICU stay he was transfused with fresh frozen plasma to correct serum proteins. He was found to have low platelets at this time. Few days later his serum creatinine was found to be $3.2 \mathrm{mg} / \mathrm{dl}$ with the urine showing protein ++ and 100 to 150 of RBC per HPF, but his urine output remained normal. Renal scanning revealed increased echogenic normal sized kidneys. By day 5 to 6 he could be weaned off from the ventilator and repeat chest $x$ ray showed clearance of shadows.

On day seven he was found to be drowsy and developed a right side face- arm- leg weakness, and the CT head showed multiple bilateral hemorrhagic 
patches with cerebral edema. The serial blood counts from admission to the onset of neurological signs revealed a decline in hemoglobin from $17 \mathrm{mg} / \mathrm{dl}$ to 9.2 $\mathrm{mg} / \mathrm{dl}$, platelet from $85 \times 10 \%$ to $55 \times 10^{\%} / \mathrm{L}$, white cell count from $22 \times 10^{\%} / \mathrm{L}$ to $11.8 \times 10^{\%} / \mathrm{L}$. His platelet counts which had been stabilized around $80 \times 10 \%$, started to drop rapidly. His bleeding time was $2 \mathrm{~min}$, clotting time 3min, INR for prothombin time 1.15 with the blood picture revealing anemia and thrombocytopenia. However fragmented red cells were not seen. His reticulocyte count was $0.5 \%$ with the direct coombs and cold agglutinin tests negative. His Ddimer levels were normal. His LDH levels were 2941 $U / L$ which was 6 times higher than the upper normal. With the clinical suspicion of TTP, we started plasma exchange on a daily basis for one week and then every other day for another week. The patient showed marked clinical improvement and the platelet count rose to $194 \times 10^{9}$ by the end of the cycles and the LDH levels came down. He recovered from the neurological weakness without any residual effect. Repeat mycoplasma IgM antibodies were positive with a titer of $1 / 80$.

\section{Discussion}

TTP is primarily seen in adults, with an incidence of 4 to 11 cases per million people in United States ${ }^{9}$. The underlying pathology is the deficiency of Von Willebrand Factor cleaving protease, named ADAMTS13. This will lead to large multimers of Von Willebrand factor, interacting with platelets, forming fibrin and platelet rich thrombi resulting in TTP ${ }^{10}$. ADAMTS13 may be deficient due to inherent deficiency or circulating inhibitors. Infections triggering antibody response against this protease is thought to be the mechanism of TTP of infectious etiology ${ }^{11}$. Mycoplasma is known to induce an inappropriate immune response, which explains most of the extrarespiratory complications such as cold autoimmune hemolytic anemia, but antibodies against ADAMTS13 are not yet demonstrated in mycoplasma.

We made the diagnosis of TTP as he showed the pentad of clinical features of TTP and could exclude alternative explanations for the given presentations, such as disseminated intravascular coagulation (DIC), hemolytic uremic syndrome (HUS), dengue shock syndrome, tuberculosis, Legionnaire's disease, hemophagocytic syndromes as discussed below and retrospectively, because he recovered with the institution of plasma exchange therapy.

Schistocytes to suggest MAHA were not detected in his blood film but MAHA can be diagnosed in the absence of schistocytes ${ }^{15}$. The very high LDH levels and rising SGOT levels (from $109 \mathrm{U} / \mathrm{L}$ to $439 \mathrm{U} / \mathrm{L}$ ) compared to SGPT levels (from $335 \mathrm{U} / \mathrm{L}$ to $192 \mathrm{U} / \mathrm{L}$ ) without a liver disease, coupled with anemia, in the absence of bleeding or muscle damage, suggested hemolysis. Negative coombs test and the absence of cold agglutinin pointed towards a non-immune mediated hemolysis, such as MAHA. But the expected rise in reticulocyte count was not seen in this case. Possible explanations could be bone marrow suppression due to sepsis, and under nutrition. This adult came with microangiopathic anemia and thrombocytopenia developing in the setting of an acute ill health; septic shock with cardio respiratory arrest. DIC was excluded as the clotting profile remained normal. Absence of high blood pressure and renal impairment needing dialysis made atypical HUS unlikely in an adult patient ${ }^{11}$. Development of neurological signs and rapid clinical improvement with plasma exchange supports TTP rather than HUS. Dengue shock syndrome which was the leading infectious cause that can present in a similar fashion was unlikely due to several reasons. Most dengue cases have leucopenia often below $4 \times 10^{9}$ when presenting in a shock stage. This degree of anemia in dengue is most likely due to bleeding, which was not seen in this case. Furthermore dengue hemorrhagic fever often presents with platelet counts much lower than this and dengue serology was negative ${ }^{12}$. Absence of organomegaly and lymphadenopathy and normal coagulation made hemophagocytic syndrome unlikely.

This patient had neurological signs and multiple minute hemorrhagic foci in the CT brain which was not seen in subsequent brain imaging. In most of the adult TTP cases brain imaging is normal, in spite of neurological signs. Reports of minute multifocal ischemia and hemorrhagic patches were reported in some other cases $^{13}$.

The rise of Mycoplasma IgM titer from negative to $1 / 80$ within 5 days together with the chest shadows, suggested mycoplasma pneumonia. There was no evidence of other bacterial pneumonias with the blood, endotracheal tube or urine cultures. Legionnaire's pneumonia presents in a similar fashion including gastro intestinal symptoms, and generalized neurological signs and responds to clarithromycin which was given to this patient. In its classic presentations there would not be a neutrophilia and focal neurological signs are rare ${ }^{14}$.

Previously published case reports on the association between TTP and mycoplasma had some similarity to our case. All the cases initially presented with prominent respiratory symptoms, and later developed the neurological and hematological manifestations of TTP. The gap between the onset of respiratory symptoms and appearance of neurological signs was 7-9 days in our case, where it was only 4 days in a previous report? ${ }^{7}$. This gap probably indicates the time for immune activation for the causation of TTP. 
The treatment of choice for TTP is plasma exchange with an exchange volume of 1 to 1.5 times the estimated plasma volume of the patient on a daily basis until the platelet count increases to above $150 \times 10^{9}$. Plasma infusion which also has shown to be effective is inferior to plasma exchange ${ }^{3}$. Glucocorticoids are indicated in the idiopathic variety, in non responders and in relapses ${ }^{1}$. This patient did not receive steroids, but showed clinical improvement with the initial few days of plasma exchange, and platelet counts started to rise after a week of regular plasma exchange. The plasma exchange was performed every other day for another week and the counts rose to $194 \times 10^{9} / \mathrm{L}$. The LDH level, which is a monitoring marker of TTP treatment ${ }^{11}$, came down from $2941 \mathrm{U} / \mathrm{L}$ to $1131 \mathrm{U} / \mathrm{L}$ within a five day period. Thereafter the patient was observed in the medical ward and arranged a follow up plan to track relapses.

\section{Conclusions}

Thrombotic thrombocytopenic purpura may be an uncommon complication of mycoplasma pneumoniae, and needs further studies to elucidate the exact nature of the causative association. However, early recognition of TTP and initiation of plasma exchange therapy seems life saving.

\section{References}

1. Allford SL, Hunt BJ, Rose P, Machin S. Guidelines on the diagnosis and management of the thrombotic microangiopathic haemolytic anaemias. British Journal of Haematology 2003; 120: 556-73.

2. Moschowitz E. Hyaline thrombosis of the terminal arterioles and capillaries: A hitherto undescribed disease. Proceedings of New York Pathology Society 1924; 24: 21-4.

3. Rock, KH Shumak, NA Buskard. Comparison of plasma exchange with plasma infusion in the treatment of thrombotic thrombocytopenic purpura. New England Journal of Medicine 1991; 325: 393-397.
4. Vesely SK, George JN, Lammle B. ADAMTS13 activity in thrombotic thrombocytopenic purpura-hemolytic uremic syndrome: relation to presenting features and clinical outcomes in a prospective cohort of 142 patients. Blood 2003; 102: 60-8.

5. Zheng XL, Kaufman RM, Goodnough LT, Sadler JE. Effect of plasma exchange on plasma ADAMTS13 metalloprotease activity, inhibitor level, and clinical outcome in patients with idiopathic and nonidiopathic thrombotic thrombocytopenic purpura. Blood 2004; 103: 4043-49.

6. Bar Meir E, Amital H, Levy Y, Kneller A, Bar-Dayan Y, Shoenfeld Y. Mycoplasma-pneumoniae-induced thrombotic thrombocytopenic purpura. Acta Haematologica 2000; 103(2): 112-5.

7. Cameron D, Welsby P, Turner M. Thrombotic thrombocytopenic purpura due to Mycoplasma pneumoniae. Postgraduate Medical Journal 1992; 68 (799): 393-4.

8. Jayantha UK. Mycoplasma pneumoniae infection in Sri Lanka. Sri Lanka Journal of Child Health 2007; 36: 43-7.

9. Terrell DR, Williams LA, Vesely SK, Lammle B, Hovinga JA, George JN. The incidence of thrombotic thrombocytopenic purpura-hemolytic uremic syndrome: all patients, idiopathic patients, and patients with severe ADAMTS-13 deficiency. Journal of Thrombosis and Haemostasis 2005; 3:1432-6.

10. Moake JL. Thrombotic microangiopathies. New England Journal of Medicine 2002; 347: 589-600.

11. Han-Mou Tsai. Advances in the Pathogenesis, Diagnosis, and treatment of thrombotic thrombocytopenic purpura. Journal of American Society of Nephrology 2003; 14: $1072-81$.

12. WHO. Dengue haemorrhagic fever: diagnosis, treatment, prevention and control. 2nd edition. Geneva 1997.

13. Bakshi R, Shaikh ZA, Bates VE, Kinkel PR. Thrombotic thrombocytopenic purpura: brain CT and MRI findings in 12 patients. Neurology 1999; 52(6): 1285-8.

14. Lane G, Ferrari A, Dreher HM: Legionnaire's disease: a current update. Medsurg Nurs 2004; 13(6): 409-14.

15. Fava, S, Galizia, AC. Thrombotic thrombocytopenic purpuralike syndrome in the absence of schistocytes. British Journal of Haematology 1995; 89: 643. 